\title{
Image guided near-infrared spectroscopy of breast tissue in vivo using boundary element method
}

\author{
Subhadra Srinivasan \\ Dartmouth College \\ Thayer School of Engineering \\ Hanover, New Hampshire 03755
}

\section{Colin M. Carpenter}

Stanford University

School of Medicine

Department of Radiation Oncology

Stanford, California 94305

\section{Hamid R. Ghadyani}

Dartmouth College

Thayer School of Engineering

Hanover, New Hampshire 03755

Senate J. Taka

Dartmouth College

Department of Computer Science

Hanover, New Hampshire 03755

Peter A. Kaufman

Dartmouth Hitchcock Medical Center Department of Hematology/Oncology One Medical Center Drive

Lebanon, New Hampshire 03756

Roberta M. DiFlorio-Alexander

Dartmouth Hitchcock Medical Center Department of Radiology

One Medical Center Drive

Lebanon, New Hampshire 03756

\section{Wendy A. Wells}

Dartmouth Hitchcock Medical Center

Department of Pathology

One Medical Center Drive

Lebanon, New Hampshire 03756

\section{Brian W. Pogue}

Keith D. Paulsen

Dartmouth College

Thayer School of Engineering

Hanover, New Hampshire 03755

\begin{abstract}
We demonstrate quantitative functional imaging using imageguided near-infrared spectroscopy (IG-NIRS) implemented with the boundary element method (BEM) for reconstructing 3-D optical property estimates in breast tissue in vivo. A multimodality MRI-NIR system was used to collect measurements of light reflectance from breast tissue. The BEM was used to model light propagation in 3-D based only on surface discretization in order to reconstruct quantitative values of total hemoglobin $(\mathrm{HbT})$, oxygen saturation, water, and scatter. The technique was validated in experimental measurements from heterogeneous breast-shaped phantoms with known values and applied to a total of seven subjects comprising six healthy individuals and one participant with cancer imaged at two time points during neoadjuvant chemotherapy. Using experimental measurements from a heterogeneous breast phantom, BEM for IG-NIRS produced accurate values for $\mathrm{HbT}$ in the inclusion with a $<3 \%$ error. Healthy breast tissues showed higher HbT and water in fibroglandular tissue than in adipose tissue. In a subject with cancer, the tumor showed higher HbT compared to the background. $\mathrm{HbT}$ in the tumor was reduced by $9 \mu \mathrm{M}$ during treatment. We conclude that 3-D MRI-NIRS with BEM provides quantitative and functional characterization of breast tissue in vivo through measurement of hemoglobin content. The method provides potentially complementary information to DCE-MRI for tumor characterization. ( 2010 Society of Photo-Optical Instrumentation Engineers. [DOI: 10.1117/1.3499419]
\end{abstract}

Keywords: optical tomography; breast cancer; image-guided; boundary element method; near infrared; clinical.

Paper 10045SSR received Feb. 1, 2010; revised manuscript received May 27, 2010; accepted for publication May 28, 2010; published online Dec. 17, 2010.

\section{Introduction}

Breast magnetic resonance imaging (MRI) is increasingly being used for screening of the high-risk population, ${ }^{1,2}$ detection and diagnosis of breast cancer, ${ }^{3}$ and monitoring of neoadjuvant

Address all correspondence to: Subhadra Srinivasan, Dartmouth College, Thaye School of Engineering, 8000 Cummings Hall, Hanover, New Hampshire, 03755, United States of America, Tel: 603-646-2119; Fax: 603-646-3699. E-mail: subhadra.srinivasan@dartmouth.edu. chemotherapy treatment (NACT) of locally advanced disease. ${ }^{4-6}$ In breast cancer screening, the advantage of conventional contrast-enhanced (CE) MRI is high sensitivity; but in the diagnostic setting the technique is limited by its specificity (ranging from 37 to $97 \%))^{7,8}$ The specificity of MRI has recently been improved with the use of dynamic CE-MRI (DCE-MRI) and MR spectroscopy. ${ }^{8}$ DCE-MRI images the breast following

$1083-3668 / 2010 / 15(6) / 061703 / 8 / \$ 25.00$ C 2010 SPIE 
gadolinium injection and provides kinetic parameters such as signal enhancement ratio, contrast wash-in transfer constant ( $\left.K_{\text {trans }}\right)$, contrast wash-out rate constant $\left(K_{\text {ep }}\right)$, relative blood flow, relative blood volume, and mean transit time. Proton MR spectroscopy is also functional, based on detecting elevated levels of total choline compounds (tCho) and increased water by measuring water-to-fat (W-F) ratios. These techniques have recently been extended to assess the response of primary breast cancer to NACT of locally advanced disease..$^{9,10}$ The metrics used to differentiate response include tumor volume ${ }^{5}$ change in tCho ${ }^{11}$ and $\mathrm{W}-\mathrm{F}$ ratio ${ }^{12}$ using MR spectroscopy, signal enhancement ratio $^{13}$ and other kinetics ${ }^{14,15}$ through contrast MRI. A potential drawback associated with MRI is the inability to provide quantitative and absolute values of physiological parameters. Here, we present a multimodality paradigm that combines optical techniques with MRI to provide absolute and quantitative values of HbT, oxygen saturation, water, and scatter that is repeatable and can be used for breast tissue characterization and evaluation of response to NACT.

Optical technologies using near-infrared (NIR) light (in the wavelength range of 600-1000 nm) provide measurements of HbT noninvasively. ${ }^{16}$ The data are obtained using a probe consisting of optical fibers suitably placed around the periphery of the breast, which transmit laser light at low power and collect measurements of light reflectance. These measurements are then analyzed with a model of light propagation in tissue to provide maps of absorption and scattering coefficients. From these optical properties, HbT and other associated parameters can be obtained by spectral fitting. Several studies have investigated the use of stand-alone NIR imaging and spectroscopy for breast cancer diagnosis and showed intrinsic tumor-to-normaltissue contrasts of up to $200 \%$ in HbT. ${ }^{17-20}$ In a cohort of 100 subjects imaged optically, a statistically significant difference was found between malignant and normal tissue. ${ }^{21}$ The resolution of NIR imaging by itself is poor, confounded by the high optical scattering of tissue. It has been improved in recent studies through a multimodality approach, ${ }^{22-24}$ where a combined MRI-NIR method provides high-resolution quantitative and absolute functional characterization of tissue in vivo. Such an imaging technique uses MRI anatomical structure to guide the optical parameter recovery. The results complement DCE-MRI by giving additional tissue vascular and oxygenation information in vivo that is quantitative and repeatable.

Prior studies have applied this technique to recover 2-D maps of NIR estimates during clinical breast exams in vivo. ${ }^{24,25}$ However, it is well known that light propagation in tissue is inherently a 3-D process and 2-D approximations may result in artifacts or erroneous values. ${ }^{26} 3$-D problems are plagued by long computational times resulting from the need to create volumetric meshes of arbitrary tissue shapes and reconstruct optical parameters for large numbers of unknowns. The number of parameter estimates can be reduced by using "hard priors," where the number of unknowns is proportional to the number of known homogeneous regions [termed as image-guided NIR spectroscopy (IG-NIRS) $].{ }^{27}$ However, traditional techniques based on the finite element method (FEM) still require volumetric meshing, which can be time consuming and unreliable and difficult to automate. The boundary element method (BEM) reduces the dimensionality of the computations through surface discretization (rather than requiring a volume mesh in three dimensions). The method was found to be $44-72 \%$ computationally faster, but more importantly, it is more reliable because surface meshing is easily accomplished and robust and more easily automatable. ${ }^{28}$ Previous studies using BEM have been limited to simulations and experiments. ${ }^{28}$ Here, we extend the functionality of BEM for 3-D multispectral IG-NIRS and provide the first experimental validation and preliminary 3 -D clinical breast imaging results in vivo. We present data from six healthy subjects and a subject with cancer at two different time points during neoadjuvant chemotherapy. The results illustrate that BEM provides quantitative NIR property parameter estimates that allow reliable 3-D volumetric breast tissue characterization that has the potential to complement results obtained from MRI.

\section{Methods}

\subsection{MRI Imaging Studies}

A 3T Philips MR scanner was used to acquire T1-weighted and DCE-MR scans. 2-D T1-weighted Spin Echo (TR/TE = 900/10, flip angle $=90 \mathrm{deg}$ ) images were obtained for all subjects. For the subject with abnormality, regions of interest, determined by DCE-MR, were recorded by injecting a bolus of contrast agent (Magnavist) intravenously and taking a series of 3-D T1$\mathrm{W}$ volume images $(\mathrm{TR} / \mathrm{TE}=10 / 6$, flip angle $20 \mathrm{deg})$ after each minute, beginning $40 \mathrm{~s}$ postinjection.

\subsection{NIR Imaging Studies}

The NIR instrumentation is shown in Fig. 1 and used 16 fibers to sequentially transmit light from each of multiple laser sources and deliver the measured signal to a bank of photomultiplier tube detectors. Light from six individual diode lasers (wavelength ranges of $660-850 \mathrm{~nm}$ ) was sequentially delivered through the fibers to the breast with an intensity modulation frequency of $100 \mathrm{MHz}$. The amplitude and phase shift of the transmitted light were recorded at multiple locations around the periphery of the breast. These frequency domain measurements allow separation of absorption and scatter processes during image reconstruction. The fiber-optic cables were nonmagnetic and integrated with a MR breast coil, allowing NIR data acquisition while the scanner was in operation. The patient was positioned prone inside the MRI (3T Philips Achieva, X-series) with the breast pendant into the fiber-holder interface. Although much of our initial work positioned the fibers in a circular geometry, ${ }^{29}$ a version of the system that conforms to the standard parallel-plate biopsy geometry ${ }^{27}$ is also available to facilitate clinical workflow. This change in geometry does not significantly alter the NIR imaging performance.

\subsection{Image Segmentation and Breast Surface Rendering}

The MR images were segmented into adipose and fibroglandular (FG) tissues using the T1-weighted MR acquisitions based on thresholding and region-growing operations applied to the grayscale intensities. The image segmentation was accomplished using a commercial software package (Mimics ${ }^{\mathrm{TM}}$, Materialise Inc.). An example of this process is shown in Fig. 2. In the subject with cancer, the corresponding DCE-MRI was used to segment the lesion. The segmented tissues were surface rendered using 


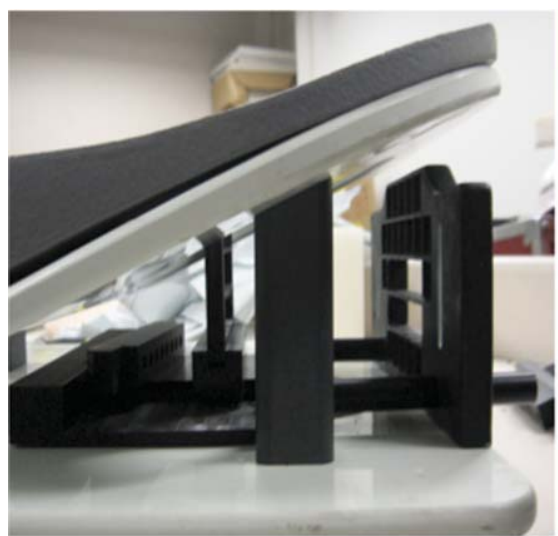

(a)

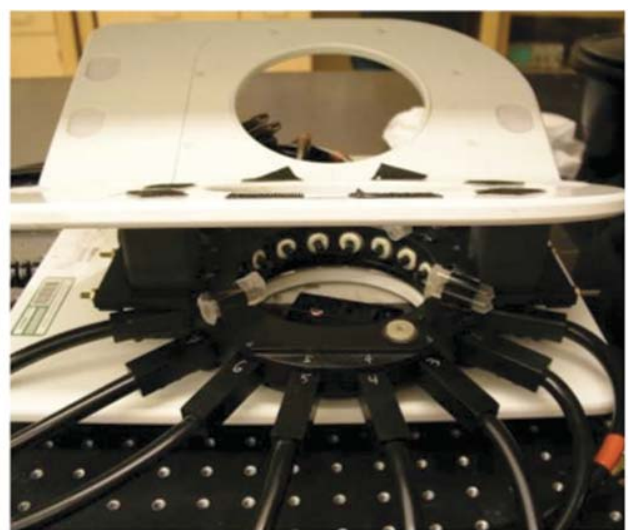

(b)

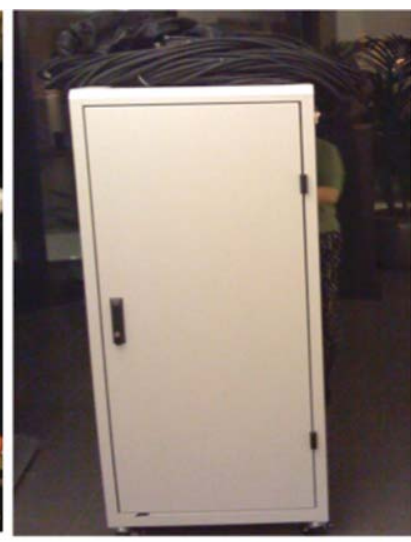

(c)

Fig. 1 (a) Biopsy plate slab interface with slots for fibers, (b) circular breast interface containing optical fibers shown positioned in the MR breast coil, and (c) integrated mobile cart containing the NIR data collection instrumentation.

the software and exported to apply diffusion light modeling for image reconstruction.

\subsection{BEM-Based Multispectral Image Reconstruction}

The diffusion approximation to radiative transport theory has been successfully used in the past to model light propagation in highly scattering media. ${ }^{30-32}$ The BEM was applied to the diffusion approximation under the assumption that the tissue being imaged contains homogeneous regions whose boundaries are known a priori (from MRI). ${ }^{28,33}$ It reduces the dimensionality of the problem, requiring only surface rather than volume discretization in three dimension, which is significantly easier and more reliable to generate. Using this numerical model to solve the diffusion equation, unknown NIRS parameters can be reconstructed from boundary measurement of light amplitude and phase. This inverse problem was solved iteratively using a modified Newton's method and Levenberg-Marquardt regularization. ${ }^{34}$

By applying a BEM model for the diffusion equation, average region-based IG-NIRS estimates of HbT, oxygen saturation, water, and scatter were obtained for each of the constituent tis- sues in the breast. Briefly, the acquired boundary measurements of light amplitude and phase underwent a calibration procedure to compensate for system offsets due to source-detector fiber transmission, alignment characteristics, and errors in discretization or model-data mismatch. ${ }^{35}$ This calibration procedure, also called homogeneous fitting, used a two-step procedure to estimate the slopes of the log of light intensity and phase with distance $r$ using (i) analytical solution to an infinite medium and (ii) Newton-Raphson fitting with a homogeneous BEM model. Postcalibration, the clinical image reconstructions were accomplished using a multispectral direct chromophore and scatter recovery algorithm that has been demonstrated to reduce cross-coupling between parameters and provide the most accurate optical estimates of tissue composition. ${ }^{36,37}$ In this reconstruction, concentrations of oxyhemoglobin ( $\mathrm{HbO})$, deoxyhemoglobin $(\mathrm{Hb})$, water, and scatter were obtained, and extended indices such as $[\mathrm{HbT}]=[\mathrm{Hb}]+[\mathrm{HbO}]$, and oxygen saturation $\mathrm{S}_{\mathrm{t}} \mathrm{O}_{2}=[\mathrm{HbO}] /[\mathrm{HbT}]$ in percent were calculated. The starting values for the reconstruction were obtained from the homogeneous fitting procedure, except for water whose starting value was set at $100 \%$. This recovered the most accurate water content in simulations and experiments. The reconstruction constrained water content to be within the range $0-100 \%$.

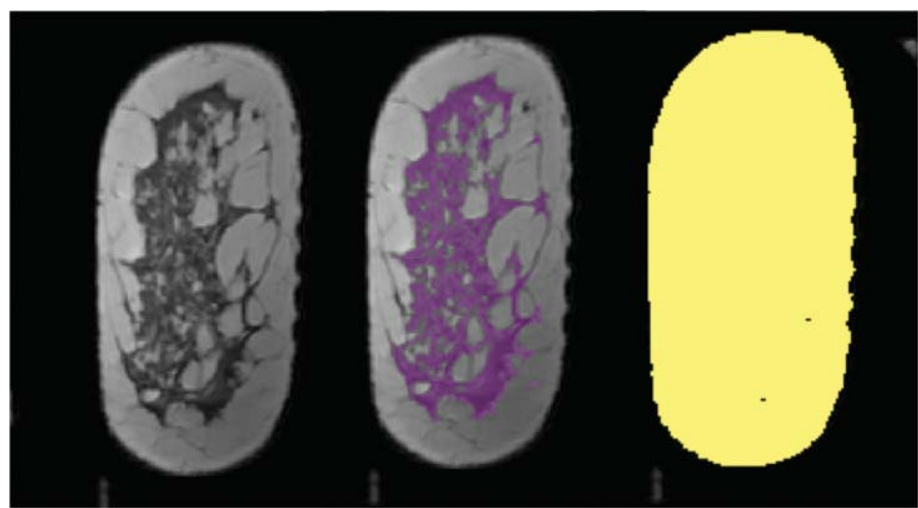

(a) (b)

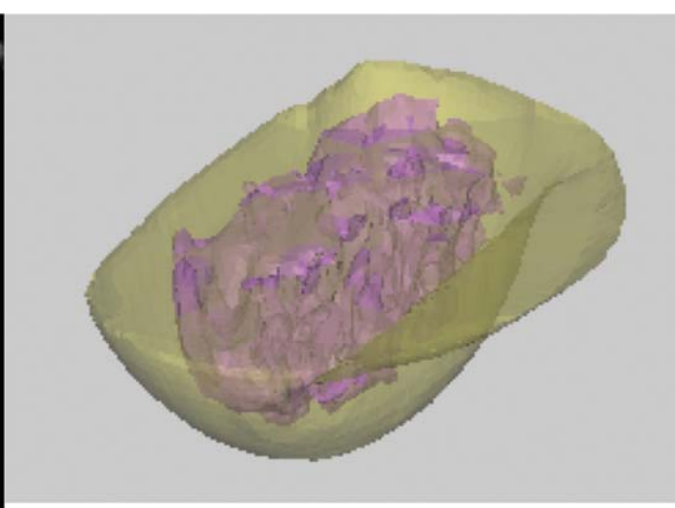

(d)

Fig. 2 (a) Sample MR coronal image of a healthy subject, (b) thresholding results in a binary mask delineating the FG tissue from the rest of the breast shown in purple, and (c) binary mask delineating the breast from the background. Surface renderings for the breast outer contour and FG tissue from 3-D segmentation are shown in (d). This geometry was directly used in the BEM toolbox for NIR image reconstruction. 


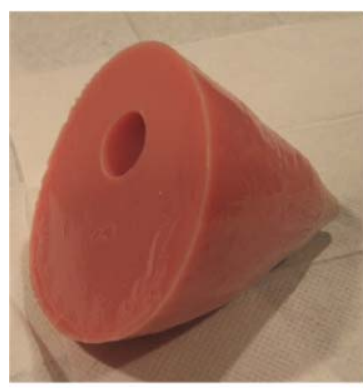

(a)

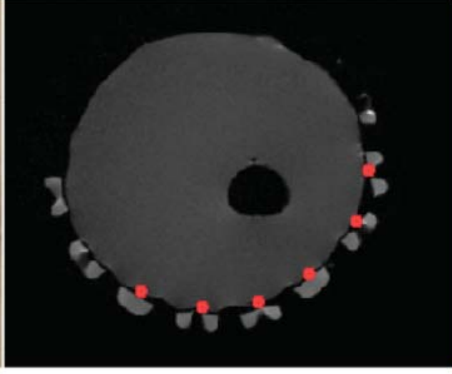

(b)

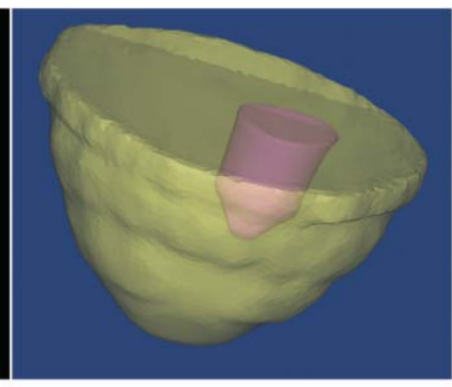

(c)

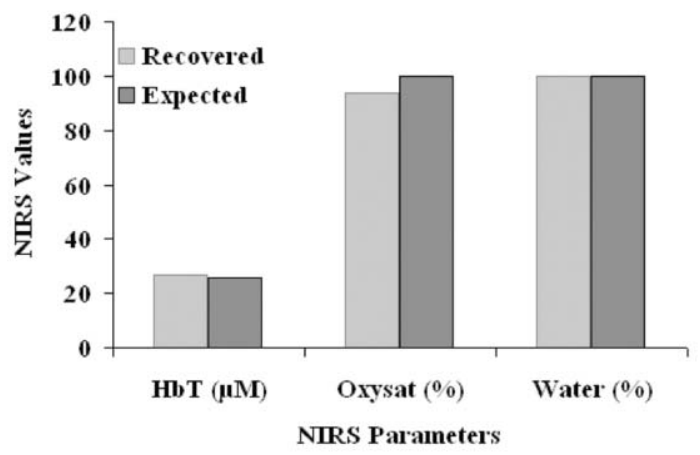

(d)

Fig. 3 (a) Breast phantom imaged in the MRI-NIR system is shown with a representative MR slice in (b) containing fiducial markers indicating the positioning of the fibers (in red) and (c) segmented background and inclusion surfaces. These surfaces were used to reconstruct the NIRS parameters of the phantom shown in (d) with comparison to expected values. Water was reconstructed with $100 \%$ accuracy in this case, because it was the maximum allowable by the reconstruction.

\subsection{Patients}

The clinical enrollment consisted of seven patients of whom six were healthy subjects with no known abnormality, and one was diagnosed with infiltrating ductal carcinoma (IDC). All subjects provided informed consent and were imaged according to study protocols approved by the Institutional Review Board at Dartmouth. NIR measurements were acquired simultaneously with MRI acquisition in all subjects with no additional increase in imaging time. Up to six wavelengths were applied during the NIR exam. The MRI of the subjects was used to provide anatomical tissue structure for optical image reconstruction.

The subject with cancer was a 36-year-old female volunteer diagnosed with a 3-cm, intermediate-grade, triple-negative (for estrogen, progesterone, and HER2/neu expression), nodepositive IDC with three satellite malignancies in her left breast. She underwent neoadjuvant chemotherapy, and her chemotherapy regimen consisted of six cycles of docetaxol/adriamycin/ cylcophosphamide. The subject had a complete pathological response subsequent to chemotherapy as confirmed by histology. Multiple imaging sessions of T1-weighted and DCE-MRI and NIR measurements were performed over the course of treatment. The first imaging session occurred 1 day prior to her first cycle of chemotherapy (cycle 1) in the circular geometry. Imaging sessions thereafter were performed within $48 \mathrm{~h}$ of chemotherapy for cycles 2 and 4 and postchemotherapy, prior to surgery. Out of these four imaging sessions, the plane of the NIR fibers in session 1 was offset from the lesion sampled in sessions 2 and 3 by $5 \mathrm{~mm}$. The plane of fibers was consistent in sessions 2 and 3 , and optical data from these visits (labeled 1 and 2 in results) were used to reconstruct the IG-NIRS estimates for background and tumor tissues. In session 4, the tumor was no longer visible in the MRI and hence is not shown here.

\section{Results}

\subsection{Experimental Validation in a Breast-Shaped Phantom}

A gelatin phantom was created using a breast mold fabricated from a segmented breast MR volume. Pig blood was added to the phantom during preparation to cause absorption due to hemoglobin and titanium dioxide $\left(\mathrm{TiO}_{2}\right)$ was added to introduce scattering. ${ }^{38}$ By measuring the hematocrit of the blood and converting to $\mathrm{HbT}$, the mixture represented a $14-\mu \mathrm{M}$ concentration. A cylindrical tube of $30 \mathrm{~mm}$ diam filled with a $26-\mu \mathrm{M}$ solution was inserted into the gelatin to create an inclusion that presented a total hemoglobin contrast of $\sim 2: 1$. A photograph of the phantom is shown in Fig. 3(a). A homogeneous cylindrical phantom was also made from the same gelatin mixture and utilized for data calibration. The breast phantom with inclusion was imaged optically using six wavelengths, and MR images of the phantom were acquired with fiducial markers to denote fiber locations; a cross section is shown in Fig. 3(b). The MRI was segmented to create 3-D surfaces of the breast and inclusion as shown in Fig. 3(c). These surfaces were used along with optical frequency domain measurements to reconstruct the NIRS parameters for the background and the inclusion. The recovered values in the inclusion are compared to the expected values in Fig. 3(d). The results show that the error in recovered HbT was $<3 \%$, illustrating that quantitative characterization is possible 
(a)

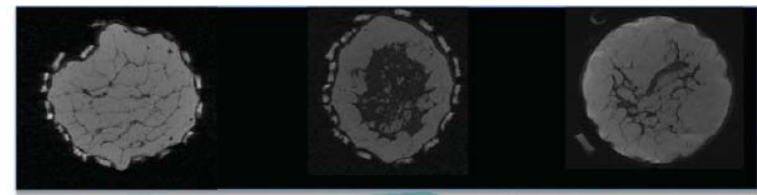

(b)

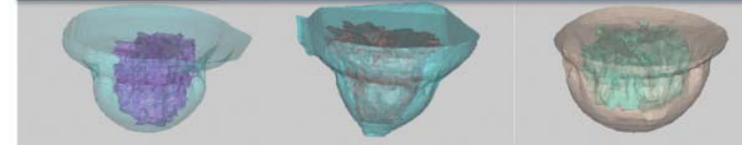

(c)

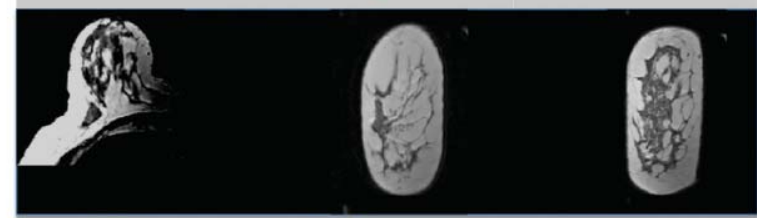

(d)

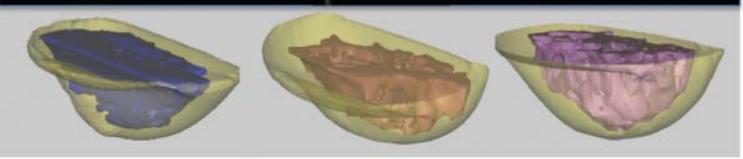

(e)

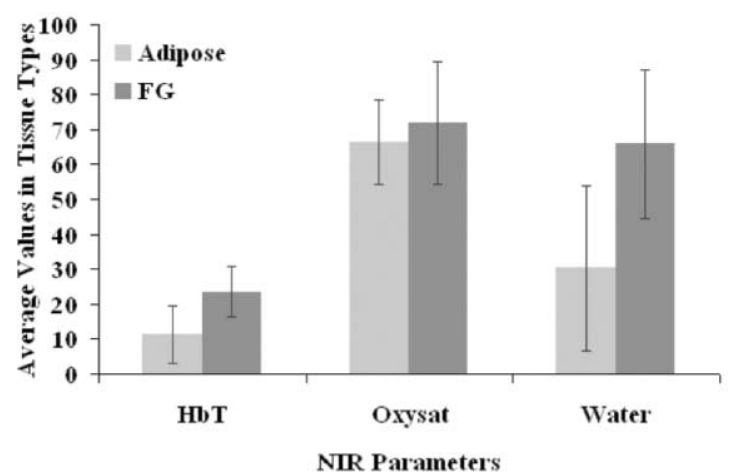

Fig. 4 (a) Representative MRI coronal slices are shown for healthy subjects 1-3 and (b) corresponding surfaces for adipose and fibroglandular (FG) tissues obtained from MR image segmentation. (c) Same as (a) for healthy subjects 4-6 imaged in the slab-geometry and (d) corresponding surfaces. Reconstructed estimates of adipose and FG are shown in (e) the bar graph with standard deviation for these six subjects.

with BEM based on experimental measurements. The error in oxygen saturation was $6.3 \%$.

\subsection{Healthy Breast Tissue Characterization of Adipose and FG Tissue Types}

For healthy breast characterization, the MRI of each subject was segmented into its adipose and FG tissue structures, and then surface rendered using Mimics ${ }^{\mathrm{TM}}$. These surfaces along with a representative MR image for these subjects are shown in Fig. 4. They were input as the geometry for IG-NIRS image reconstruction. All NIR parameters were updated during the iterative reconstruction procedure. Using the BEM spectral reconstruction with starting values obtained from calibration and the measured data, IG-NIRS estimates were recovered for adipose and FG tissues of the breast. The reconstructed results are shown in Fig. 4(b). The data show that the average $\mathrm{HbT}$ in FG tissue is higher than that of adipose tissue. A similar trend was observed in water content with FG tissue containing higher water, when compared to adipose tissue. Oxygen saturation was slightly increased in FG $(72 \% \pm 17 \%)$ compared to adipose $(66 \% \pm 12 \%)$ tissue. Statistical analysis using paired t-test showed a $p$-value of 0.062 for difference in HbT, $p$-value of 0.34 for oxygen saturation and $p$-value of 0.054 for difference in water. The $p$-values for $\mathrm{HbT}$ and water are close to being statistically significant ( $p$-value $=0.05$ ) ; however due to the limited sample size and variation in number of wavelengths used, it is difficult to draw conclusions from this population. In the individual subjects, every subject showed higher $\mathrm{HbT}$ and water in FG as compared to adipose tissue. Scattering estimates were also reconstructed, but an insufficient number of wavelengths were available to determine their accuracy.

The reconstructions converged in four to six iterations, and convergence was deemed when the change in projection error (least-squares difference between measured and model data) was $<0.5 \%$ between successive iterations. The normalized projection error did not drop below 0.5 for clinical data, probably because of increased noise in the measurements and the greater approximation in the data-model match in vivo. The experimental projection error in phantoms dropped to 0.25 in comparison.

\subsection{Imaging Subject with Cancer}

The T1-weighted MRI from each session was used to segment the adipose and FG tissues and the corresponding DCE-MRI was used to segment the lesions in a semi-automatic way with guidance from radiologist. The segmented surfaces are displayed in Fig. 5 along with the imaging plane denoted by the fiber locations (in red).

Following data calibration, the IG-NIRS estimates were reconstructed for adipose, FG, and tumor tissue and are presented in Fig. 5(b) for HbT. The results show a reduction in the tumor hemoglobin from visit 1 to visit 2. The tumor $\mathrm{HbT}$ was higher than the HbT of background tissues (adipose and FG) as well as higher than the averages from the healthy subjects. A reduction in the adipose and FG $\mathrm{HbT}$ was also observed in visit 2 relative to visit 1 , indicating that both the background and tumor vasculature have undergone change during treatment. Oxygen saturation was found to decrease in all tissues, and no noticeable trend was observed in water content.

\section{Discussion}

The combined MRI-NIR imaging system used in this study has been optimized for clinical use and previous work in two dimensions has shown that high-resolution image-guided NIRS provides important functional information related to breast tissue vasculature and metabolic activity. ${ }^{25}$ The development of a BEM numerical model has allowed us to move toward fully automated 3-D image recovery, a major step forward in terms of processing clinical data in a systematic manner. Use of frequency-domain measurements at six wavelengths ensured adequate separation of NIR absorption and scatter and incorporation of priors from MRI ensured accurate region-based optical property estimates in the breast volume at the resolution of the segmented MR. Using this system, we have evaluated BEM results in a 3-D breast phantom and in 3-D breast volumes consisting of healthy and diseased tissues.

BEM-based reconstruction using experimental measurements obtained from a breast-shaped phantom with a $30-\mathrm{mm}$ inclusion used to mimic tumor showed that quantitative values 


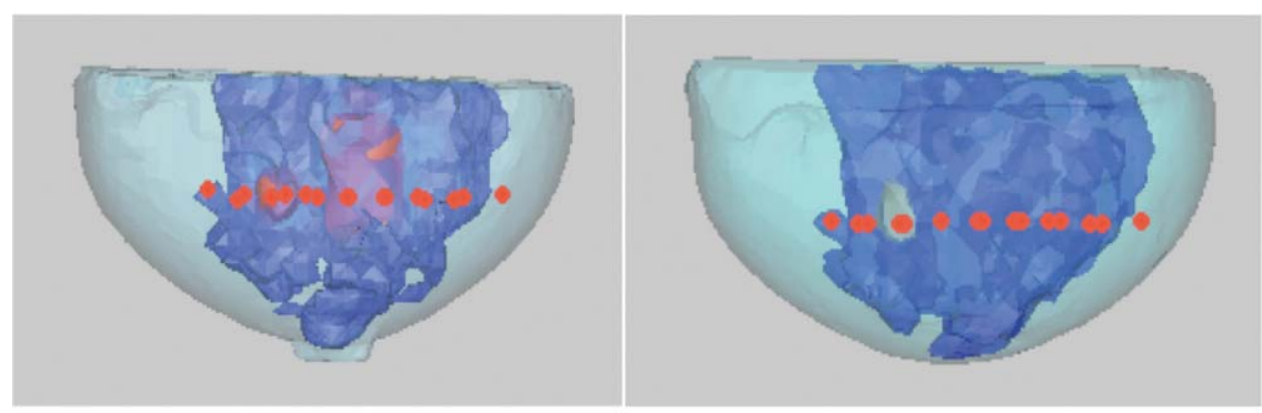

(a)

(b)

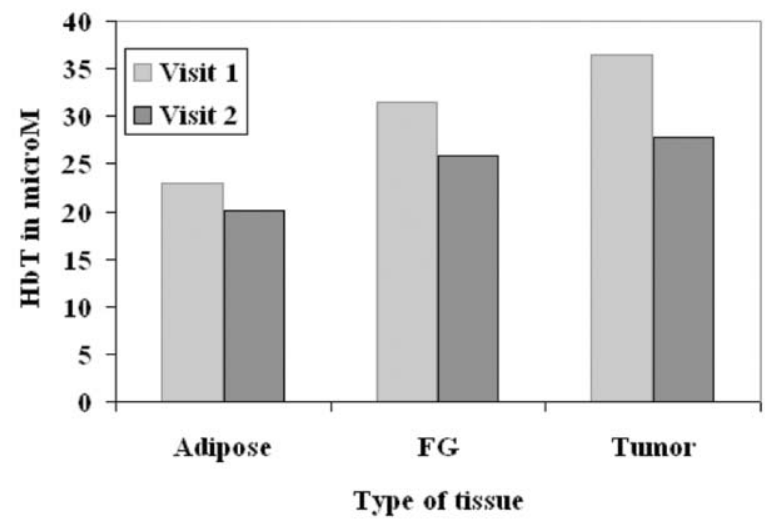

(c)

Fig. 5 Surface renderings of adipose, FG, and lesions segmented from T1-weighted MRI and DCE-MRI of subject 7 undergoing NACT are shown for (a) visit 1 corresponding to cycle 2 and (b) visit 2 corresponding to cycle 4 . The red markers indicate optical fiber locations. (c) Reconstructed IG-NIRS values are displayed for HbT in adipose, FG, and tumor tissues for both visits. The subject experienced a pathologic complete response.

for total hemoglobin could be recovered with an accuracy $>97 \%$ (Fig. 3). Oxygen saturation was quantified with 93\% accuracy, and water contrast was $100 \%$ accurate. Previous experiments using cylindrical phantoms have shown that increases in HbT can be tracked successfully, and the oxygen dissociation curve can be recovered with an accuracy of $>93 \% .{ }^{39}$

The results from healthy subjects show consistently higher total hemoglobin in FG tissue, nearly twice that of adipose (Fig. 4). This finding agrees with the expectation that more blood vessels are found in glandular tissue relative to adipose. ${ }^{40}$ The HbT values for adipose and FG tissue are comparable to those estimated in a previous study using MRI-guided NIR characterization in a 2-D circular geometry: ${ }^{24} 23.6 \pm 7.1 \mu \mathrm{M}$ as compared to $22.4 \pm 7.3 \mu \mathrm{M}$ (previously) for FG and $11.3 \pm$ $8.3 \mu \mathrm{M}$ as compared to $17.1 \pm 3.2 \mu \mathrm{M}$ (previously) for fat. The differences may arise from a combination of factors, such as intersubject variability and 2-D versus 3-D image recovery. It is well known that NIR imaging in three dimensions is more accurate than two dimensions ${ }^{26}$ since light propagation is inherently a 3-D process.

Situations where the 2-D NIR imaging plane does not contain the regions of interest necessitate the use of a 3-D image formation procedure. However, even in three dimensions, the location of the imaging plane has an impact on the recovered contrast and can result in underestimation of true contrast. Results from simulations have shown that the accuracy in HbT drops by $2.3 \%$ for every $1 \mathrm{~mm}$ of distance the NIR imaging plane is displaced from the center of the tumor. ${ }^{41}$ Thus, a fiber array placement for $10 \mathrm{~mm}$ from the center of the tumor would translate into an error of $23 \%$ in $\mathrm{HbT}$, which is a significant underestimation of contrast. This issue is currently being addressed by adding the capability of acquiring multiple planes of data to reduce the dependence on optical fiber placement.

In the subject with cancer, the higher HbT recovered in the tumor is consistent with the expectation that breast tumors associated with IDC are more vascularized than surrounding normal tissues. ${ }^{40}$ The tumor HbT was also higher than the average from the healthy tissues as well as the background values in the same breast. Indeed, studies examining relationships between $\mathrm{HbT}$ obtained from NIR tomography and histopathology measures of mean blood vessel density have shown statistically significant correlations. $^{21}$

In this subject undergoing treatment, a significant drop $(\sim 9 \mu \mathrm{M})$-nearly $25 \%$ in tumor HbT-was observed, decreasing from $36.4 \mu \mathrm{M}$ in visit 1 to $27.8 \mu \mathrm{M}$ in visit 2 (see Fig. 5). The background values for adipose and fibroglandular tissues were reduced as well. Traditionally, imaging modalities are used to analyze tumor response to chemotherapy, but the response of surrounding tissue is less clear. Because chemotherapy operates by killing cancer cells, it is likely to affect the extracellular matrix of surrounding cells as well, which may account for the observed changes. Previous studies have shown that breast tissue composition is affected by hormonal changes during the course of the menstrual cycle. ${ }^{17,42}$ It is possible that NACT may induce hormonal changes that alter breast composition. For example, in a recent study reviewing 420 patients treated from 1982 to 2004 with different NACT combinations, ${ }^{43} 23 \%$ of the patients had NACT-induced variations in HR status. Studying 
tissue response in the manner described here will allow us to identify whole breast composition changes during treatment.

The assumption behind BEM approach to modeling the breast tissue is that the tissue contains several homogeneous regions with well-defined boundaries. To address the heterogeneity within tissue, a coupled FEM-BEM method may be more suitable. ${ }^{44}$ Here, we have focused on quantitative bulk average values of the different types of tissues.

In summary, we have provided in vivo 3D breast tissue estimates using BEM in an IG-NIRS setting with healthy and disease tissue undergoing NACT. Further pilot and phase 1 clinical trials are ongoing. This paper demonstrates the ability to combine NIRS with MRI to provide quantitative estimates of vasculature through measurement of hemoglobin content reconstructed with a 3-D BEM approach involving only surface discretization and to supply complementary information through a multimodal imaging method.

\section{Acknowledgments}

We thank Shudong Jiang from Thayer School of Engineering for assistance in system calibration and Susan A. Schwarz from Academic Computing at Dartmouth College for software troubleshooting. This work was supported by NIH Grants No. R01 CA69544 and No. R01EB007966 and the Neukom Computational Institute at Dartmouth College.

\section{References}

1. C. D. Lehman, et al., "Screening women at high risk for breast cancer with mammography and magnetic resonance imaging," Cancer 103(9), 1898-1905 (2005).

2. M. Kriege, et al., "Efficacy of MRI and mammography for breastcancer screening in women with a familial or genetic predisposition," New Engl. J. Med. 351(5), 427-437 (2004).

3. A. Chung, R. Saouaf, K. Scharre, and E. Phillips, "The impact of MRI on the treatment of DCIS," Am. Surgeon 71, 705-710 (2005).

4. M. W. Ah-See, et al., "Early changes in functional dynamic magnetic resonance imaging predict for pathological response to neoadjuvant chemotherapy in primary breast cancer," Clin. Cancer Res. 14(20), 6580-6589 (2008).

5. S. C. Partridge, et al., "MRI measurements of breast tumor volume predict response to neoadjuvant chemotherapy and recurrence-free survival," Am. J. Roentgenol. 184(6), 1774-1781 (2005).

6. M. D. Pickles, M. Lowry, D. J. Manton, P. Gibbs, and L. W. Turnbull, "Role of dynamic contrast-enhanced MRI in monitoring early response of locally advanced breast cancer to neoadjuvant chemotherapy," Breast Cancer Res. Treat. 91(1), 1-10 (2005).

7. S. G. Orel and M. D. Schnall. "MR imaging of the breast for the detection, diagnosis, and staging of breast cancer," Radiology 220(1), 13-30 (2001).

8. L. Bartella, C. S. Smith, D. D. Dershaw, and L. Liberman, "Imaging breast cancer," Radiol. Clin. N. Am. 45, 45-67 (2007).

9. A. R. Padhani, et al., "Prediction of clinicopathologic response of breast cancer to primary chemotherapy at contrast-enhanced MR imaging: initial clinical results," Radiology 239(2), 361-374 (2006).

10. N. R. Jagannathan, et al., "Evaluation of total choline from in-vivo volume localized proton MR spectroscopy and its response to neoadjuvant chemotherapy in locally advanced breast cancer," Br. J. Cancer 84(8), 1016-1022 (2001).

11. H. M. Baek, et al., "Predicting pathologic response to neoadjuvant chemotherapy in breast cancer by using MR imaging and quantitative 1H MR spectroscopy," Radiology 251, 653-662(3) (2009).

12. M. Kumar, et al., "Monitoring the therapeutic response of locally advanced breast cancer patients: sequential in vivo proton MR spectroscopy study," J. Magn. Res. Imag. 24, 325-332 (2006).
13. K. Li, S. C. Partridge, B. N. Joe, J. E. Gibbs, Y. Lu, L. J. Esserman, and N. M. Hylton, "Invasive breast cancer: predicting disease recurrence by using high-spatial-resolution signal enhancement ratio imaging," Radiology 248(1), 79-87 (2008).

14. M. W. Ah-See, et al., "Early changes in functional dynamic magnetic resonance imaging predict for pathological response to neoadjuvant chemotherapy in primary breast cancer," Clin. Cancer Res. 14(20), 6580-6589 (2008).

15. M. D. Pickles, M. Lowry, D. J. Manton, P. Gibbs, and L. W. Turnbull, "Role of dynamic contrast-enhanced MRI in monitoring early response of locally advanced breast cancer to neoadjuvant chemotherapy," Breast Cancer Res. Treat. 91(1), 1-10 (2005).

16. B. Chance, S. Nioka, J. Zhang, E. F. Conant, E. Hwang, S. Briest, S. G. Orel, M. D. Schnall, and B. J. Czerniecki, "Breast cancer detection based on incremental biochemical and physiological properties of breast cancers: a six-year, two-site study," Acad. Radiol. 12(8), 925-933 (2005).

17. B. W. Pogue, S. Jiang, H. Dehghani, C. Kogel, S. Soho, S. Srinivasan, X. Song, S. P. Poplack, and K. D. Paulsen, "Characterization of hemoglobin, water and NIR scattering in breast tissue: analysis of intersubject variability and menstrual cycle changes relative to lesions," $J$. Biomed. Opt. 9(3), 541-552 (2004).

18. B. W. Pogue, S. P. Poplack, T. O. McBride, W. A. Wells, K. S. Osterman, U. L. Osterberg, and K. D. Paulsen, "Quantitative hemoglobin tomography with diffuse near-infrared spectroscopy: pilot results in the breast," Radiology 218(1), 261-266 (2001).

19. B. J. Tromberg, N. Shah, R. Lanning, A. Cerussi, J. Espinoza, T. Pham, L. Svaasand, and J. Butler, "Non-invasive in vivo characterization of breast tumors using photon migration spectroscopy," Neoplasia 2(1-2), 26-40 (2000).

20. S. Srinivasan, B. W. Pogue, B. Brooksby, S. Jiang, H. Dehghani, C. Kogel, W. A. Wells, S. P. Poplack, and K. D. Paulsen, "Near-infrared characterization of breast tumors in vivo using spectrally-constrained reconstruction," Technol. Cancer Res. Treat. 4(5), 513-526 (2005).

21. S. P. Poplack, T. Tosteson, W. A. Wells, B. W. Pogue, M. P. M, A. Hartov, C. Kogel, S. Soho, J. J. Gibson, and K. D. Paulsen, "Electromagnetic breast imaging: results of a pilot study in women with abnormal mammograms," Radiology 243(2), 350-359 (2007).

22. Q. Zhang, et al., "Coregistered tomographic x-ray and optical breast imaging: initial results," J. Biomed. Opt. 10(2), 024033 (2005).

23. Q. Zhu E. B. Cronin, A. A. Currier, H. S. Vine, M. Huang, N. Chen, and $\mathrm{C}$. $\mathrm{Xu}$, "Benign versus malignant breast masses: optical differentiation with US-guided optical imaging reconstruction," Radiology 237(1), 57-66 (2005).

24. B. Brooksby, B. W. Pogue, S. Jiang, H. Dehghani, S. Srinivasan, C. Kogel, T. Tosteson, J. B. Weaver, S. P. Poplack, and K. D. Paulsen, "Imaging breast adipose and fibroglandular tissue molecular signatures using hybrid MRI-guided near-infrared spectral tomography," Proc. Nat. Acad. Sci. USA 103(23), 8828-8833 (2006).

25. C. Carpenter, et al., "Image-guided optical spectroscopy provides molecular-specific information in vivo: MRI-guided spectroscopy of breast cancer hemoglobin, water \& scatterer size," Opt. Lett. 32(8), 933-935 (2007).

26. M. Schweiger and S. R. Arridge,M. Schweiger and S. R. Arridge, "Comparison of two- and three-dimensional reconstruction methods in optical tomography," Appl. Opt. 37(31), 7419-7428 (1998).

27. C. Carpenter, S. Srinivasan, B. W. Pogue, and K. D. Paulsen, "Methodology development for three-dimensional MR-guided near infrared spectroscopy of breast tumors," Opt. Express 16(22), 1790317914 (2008).

28. S. Srinivasan, B. W. Pogue, C. Carpenter, P. K. Yalavarthy, and K. D. Paulsen, Med. Phys. 34(11), 4545-4557 (2007).

29. B. A. Brooksby, S. Jiang, H. Dehghani, C. Kogel, M. Doyley, J. B. Weaver, S. P. Poplack, B. W. Pogue, and K. D. Paulsen, "Magnetic resonance-guided near-infrared tomography of the breast," Rev. Sci. Instrum. 75(12), 5262-5270 (2004).

30. T. J. Farrell, Patterson, M. S., Wilson, B. C., "A diffusion theory model of spatially resolved, steady-state diffuse reflectance for the noninvasive determination of tissue optical properties," Med. Phys. 19(4), 879-888 (1992).

31. A. Ishimaru, Wave Propagation and Scattering in Random Media, Vol. 1. Academic Press, New York (1978). 
32. S. R. Arridge, M. Schweiger, M. Hiraoka, and D. T. Delpy, "A finite element approach for modeling photon transport in tissue," Med. Phys. 20(2), 299-309 (1993).

33. J. Sikora, A. Zacharopoulos, A. Douiri, M. Schweiger, L. Horesh, S. R. Arridge, and J. Ripoll, "Diffuse photon propagation in multilayered geometries," Phys. Med. Biol. 51(3), 497-516 (2006).

34. K. D. Paulsen and H. Jiang, "Spatially varying optical property reconstruction using a finite element diffusion equation approximation," Med. Phys. 22(6), 691-701 (1995).

35. T. O. McBride, "Spectroscopic reconstructed near infrared tomographic imaging for breast cancer diagnosis, Dissertation," Thayer School of Engineering, Dartmouth College, Hanover (2001).

36. S. Srinivasan, B. W. Pogue, S. Jiang, H. Dehghani and K. D. Paulsen, "Spectrally constrained chromophore and scattering NIR tomography provides quantitative and robust reconstruction," Appl. Opti. 44(10), 1858-1869 (2005).

37. A. Corlu, R. Choe, T. Durduran, K. Lee, M. Schweiger, S. R. Arridge, E. M. Hillman, and A. G. Yodh, "Diffuse optical tomography with spectral constraints and wavelength optimization," Appl. Opt. 44(11), 2082-2093 (2005).

38. B. W. Pogue and M. S. Patterson, "Review of tissue simulating phantoms for optical spectroscopy, imaging and dosimetry," J. Biomed. Opt. 11(4), 041102 (2006).
39. S. Srinivasan, C. Carpenter, B. W. Pogue, and K. D. Paulsen, "Imageguided near infrared spectroscopy using boundary element method: phantom validation," Proc. SPIE 7171, 717103 (2009).

40. S. Thomsen and D. Tatman, "Physiological and pathological factors of human breast disease that can influence optical diagnosis," Ann. N.Y. Acad. Sci. 838, 171-193 (1998).

41. S. Srinivasan, C. Carpenter and B. W. Pogue, "Sensitivity of hemoglobin concentration on optical probe positioning in image-guided near infrared spectroscopy," in Proc. of IEEE Eng. Med. Biol. Soc. 1994-1996 (2009).

42. N. Shah, A. Cerussi, D. Jakubowski, D. Hsiang, J. Butler, and B. Tromberg, "Spatial variations in optical and physiological properties of healthy breast tissue," J. Biomed. Opt. 9(3), 534-540 (2004).

43. O. Tacca, F. Penault-Llorca, C. Abrial, M. A. Mouret-Reynier, I. Raoelfils, X. Durando, J. L. Achard, P. Gimbergues, H. Cure, and P. Chollet, "Changes in and prognostic value of hormone receptor status in a series of operable breast cancer patients treated with neoadjuvant chemotherapy," Oncologist 12(6), 636-643 (2007).

44. S. Srinivasan, B. W. Pogue and K. D. Paulsen, "A hybrid finite element-boundary element method for modeling light propagation in tissue in 3D," Biomed. Opt., OSA Tech. Dig. 1(2) 398-413 (2010). 